\title{
Refined blowups
}

\author{
D. Kaledin And A. Kuznetsov
}

\begin{abstract}
Categorical resolution of singularities has been constructed in [KL]. It proceeds by alternating two steps of seemingly different nature. We show how to use the formalism of filtered derived categories to combine the two steps into one. This results in a certain rather natural categorical refinement of the usual blowup of an algebraic variety in a closed subscheme.
\end{abstract}

\section{Introduction}

Non-commutative algebraic geometry, at least in its modern homological version, studies triangulated categories with some enhancement (for example, DG enhancement as in [Ke2]). Passing from commutative to noncommutative world, then, consist of associating such a category to any algebraic variety $X$. The usual choice is to consider the bounded derived category $\mathcal{D}^{b}(X)$ of coherent sheaves on $X$, and take its full subcategory $\mathcal{D}^{p f}(X) \subset \mathcal{D}^{b}(X)$ formed by perfect complexes. While $\mathcal{D}^{b}(X)$ contains all the geometric objects such as the structure sheaves of subvarieties $Z \subset X$, $\mathcal{D}^{p f}(X)$ better reflects the global geometry of $X$. In particular, there are notions of homological smoothness and homological properness for enhanced triangulated categories, and $X$ is smooth resp. proper if and only if so is $\mathcal{D}^{p f}(X)$. Moreover, if $X$ is smooth, $\mathcal{D}^{p f}(X)$ coincides with the whole $\mathcal{D}^{b}(X)$.

Homologically smooth and proper categories are called saturated. It is these categories that one usually studies in non-commutative geometry, and it is only to these categories that many important results apply.

If $X$ is singular, then $\mathcal{D}^{p f}(X)$ and $\mathcal{D}^{b}(X)$ are different. If $X$ is proper, then $\mathcal{D}^{p f}(X)$ is homologically proper but it is not homologically smooth. On the other hand, $\mathcal{D}^{b}(X)$ is always homologically smooth by a recent result of Lunts [L] using a surprising and deep result of Rouqier [R]; however,

D.K. is partially supported by the Dynasty Foundation award and by the Russian Academic Excellence Project "5-100".

A.K. is partially supported by RFBR grants 14-01-00416, 15-01-02164, 15-5150045 , by the Simons foundation, and by the Russian Academic Excellence Project "5-100". 
even if $X$ is proper, $\mathcal{D}^{b}(X)$ is not homologically proper. Thus working with singular varieties presents a problem.

In the usual algebraic geometry, one handles problems of this type by resolving the singularities of an algebraic variety $X$, that is, constructing a smooth variety $Y$ equipped with a proper birational map $Y \rightarrow X$. Categorical resolution of singularities introduced recently in [KL] is a noncommutative counterpart and refinement of this procedure. The goal is to embed $\mathcal{D}^{p f}(X)$ fully and faithfully into a category $\widetilde{\mathcal{D}}$ that behaves better - it is homologically smooth, homologically proper if $X$ is proper, and can be represented as an iterated extension of categories of the form $\mathcal{D}^{b}(Z)$ for smooth algebraic varieties $Z$. Such a categorical resolution has been constructed and studied in $[\mathrm{KL}]$ for all separated schemes of finite type over a field of characteristic 0 . Taking a resolution of singularities $f: Y \rightarrow X$ is the first step of the construction. However, the pullback functor $L^{\cdot} f^{*}: \mathcal{D}^{p f}(X) \rightarrow \mathcal{D}^{b}(Y)$ is fully faithful only if $X$ has rational singularities, so that in the general case, much more work is required to achieve the goal.

Let us note that one of the main points of $[\mathrm{KL}]$ is the interplay between commutative and non-commutative geometry. From the purely noncommutative perspective, aside from the fact that the resolution $\widetilde{\mathcal{D}}$ is homologically proper if $X$ is proper, probably the main application of the construction is a recent result of Efimov [E]. He uses the explicit form of the construction of $[\mathrm{KL}]$ to refine Lunts's Theorem of $[\mathrm{L}]$ and prove that $\mathcal{D}^{b}(X)$ is homologically of finite type ("homologically finitely presented" in the language of $[\mathrm{TV}])$. The fact that the homologically smooth category $\widetilde{\mathcal{D}}$ is an iterated extension of categories of the form $\mathcal{D}^{b}(Z), Z$ smooth, plays no role - it has no non-commutative interpretation.

However, it would be presumptious to expect that the current general non-commutative perspective is the final word in the subject: there might well be some subtle ways in which categories coming from smooth algebraic varieties are substantially different from general homologically smooth categories.

In fact, if anything, our present knowledge argues exactly for this there are deep purely non-commutative results that have only been proved for saturated categories coming from algebraic varieties. One example is the non-commutative Hodge-to-de Rham degeneration theorem that has been proved in [Ka1] under a certain technical assumption, and we do not have a proof in the completely general case. Another much more striking example is the Blanc-Toën Conjecture of [Ka2]: for categories of commutative origin, it has been completely proved in [Bl], while the general case is wide open. 
Thus at least at the moment, the conservative approach taken in [KL] is obviously justified: knowing that a category is an iterated extension of categories of commutative origin is quite useful, both from the practical and from the conceptual point of view.

The point of the present paper is to show that the construction of [KL] is also very natural and simple, in fact even more natural than the usual commutative blowup.

Namely, as given in [KL], the categorical resulution procedure works by alternating two main steps of completely different nature. In this paper, we restrict our attention to one sequence of step 1 followed by step 2 , and observe that the two steps can be combined into a single procedure.

More precisely, recall that the usual blowup $Y$ of an algebraic variety $X$ in an ideal sheaf $\mathcal{I}$ is expressed as $Y=\operatorname{Proj} \mathcal{A}_{\bullet}$, where

$$
\mathcal{A}_{\text {. }}=\bigoplus_{n \geq 0} \mathcal{I}^{n}
$$

is a graded algebra sheaf on $X$. However, the sheaf $\mathcal{A}$. has more structure - it is the Rees algebra of a filtered algebra sheaf (namely, the structure sheaf $\mathcal{O}_{X}$ filtered by the powers $\mathcal{I}^{n} \subset \mathcal{O}_{X}$ of the ideal sheaf $\mathcal{I}$ ). We first show that the usual Serre theorem expressing $\mathcal{D}^{b}(Y)$ in terms of graded sheaves of modules over $\mathcal{A}$. works without any changes in the filtered context, and then observe that after an elementary modification, the filtered construction gives a "partial categorical resolution" — namely, it embeds $\mathcal{D}^{p f}(X)$ fully faithfully into an iterated extension of a copy $\mathcal{D}^{b}(Y)$ and several copies of $\mathcal{D}^{b}(Z)$, where $Z=\operatorname{Spec}\left(\mathcal{O}_{X} / \mathcal{I}\right)$ is the center of the blowup.

To obtain the whole categorical resolution of singularities by this construction, we need to know how to iterate it. It can probably be done by considering sheaves equipped with several filtrations, but at present, we have not seriously pursued this. Thus we only did a very simple thing, and we try to present it as simply as possible - Section 1 contains the precise statements of the results, and Section 2 is taken up with the proofs. It turns out that a considerable simplification can be achieved by using the machinery of exact categories and filtered derived categories, so we adopt this approach. The reader who is uncomfortable with this machinery can find an alternative formulation in terms of graded modules over the Rees algebra in the beginning of Section 2. Throughout the paper, we restrict our attention to $\mathcal{D}^{b}(-)$ and completely ignore the enhancements and the "big" unbounded derived categories of quasicoherent sheaves. Taking care of either of these two points is a straightforward exercise that does not seem to require new ideas. 


\section{Statements}

Fix once and for all a Noetherian scheme $X$. Let $\operatorname{Coh}(X)$ be the abelian category of coherent sheaves of $\mathcal{O}_{X}$-modules, and let $\mathcal{D}^{b}(X)$ be its bounded derived category. Let $\mathcal{D}^{p f}(X) \subset \mathcal{D}^{b}(X)$ be the full subcategory spanned by perfect complexes. Let $\mathrm{Fl}(X) \subset \operatorname{Coh}(X)$ be the full subcategory spanned by coherent sheaves $E \in \operatorname{Coh}(X)$ that are flat over $\mathcal{O}_{X}$ (or equivalently, locally free). The category $\mathrm{Fl}(X)$ is an exact category in the sense of Quillen.

In general, $\mathcal{D}^{p f}(X)$ is not the derived category of any abelian category. However, assume from now on for simplicity that $\operatorname{Coh}(X)$ has enough flat coherent sheaves - in other words, for any $\mathcal{E} \in \operatorname{Coh}(X)$ there exists a surjective map $\mathcal{E}^{\prime} \rightarrow \mathcal{E}$ with $\mathcal{E}^{\prime} \in \mathrm{Fl}(X)$ (for example, it suffices to assume that $X$ is quasiprojective over an affine scheme). Then $\mathcal{D}^{p f}(X)$ is the bounded derived category of the exact category $\mathrm{Fl}(X)$, as in [Ke1].

Assume given a scheme $Y$ equipped with a projective map $f: Y \rightarrow X$. By definition, we have $Y=\operatorname{Proj} \mathcal{A}_{\text {. }}$, where $\mathcal{A}$. is a sheaf of graded algebras on $X$ given by

$$
\mathcal{A}_{n}=f_{*} \mathcal{O}_{Y}(n), \quad n \geq 0,
$$

with $\mathcal{O}_{Y}(n)$ being the powers of a relatively very ample line bundle $\mathcal{O}_{Y}(1)$ on $Y$. For every $n, \mathcal{A}_{n}$ is a coherent sheaf of $\mathcal{O}_{X}$-modules, and $\mathcal{A}$. is generated by $\mathcal{A}_{0}$ and $\mathcal{A}_{1}$ as an algebra sheaf.

Denote by $\mathcal{A}$.-mod the category of sheaves

$$
\mathcal{E}_{\text {. }}=\bigoplus_{i \in \mathbb{Z}} \mathcal{E}_{i}
$$

of graded $\mathcal{A}$.-modules that are locally finitely generated over $\mathcal{A}$. (in the paper, we will never consider non-graded modules over a graded algebra, so that the notation is unambiguous). Then the components $\mathcal{E}_{n}$ of every object $\mathcal{E}$. of the category $\mathcal{A}$.-mod are coherent sheaves of $\mathcal{O}_{X}$-modules, and the action map $\mathcal{A}_{1} \otimes \mathcal{E}_{i} \rightarrow \mathcal{E}_{i+1}$ is surjective for $i \gg 0$. The category $\mathcal{A}_{\text {.-mod }}$ is abelian. Denote by $\mathcal{D}^{b}\left(\mathcal{A}_{\text {. }}\right)$ its bounded derived category. Let $\mathcal{A}$.- $\bmod _{\text {tors }} \subset \mathcal{A}$.-mod be the full abelian subcategory spanned by objects $\mathcal{E}$. such that $\mathcal{E}_{i}=0$ for $i \gg 0$, and let $\mathcal{D}_{\text {tors }}^{b}\left(\mathcal{A}_{\text {. }}\right)$ be its bounded derived category. Then the subcategory $\mathcal{A}_{\mathbf{0}}-\bmod _{\text {tors }} \subset \mathcal{A}$.-mod is thick, the natural embedding $\mathcal{D}_{\text {tors }}^{b}\left(\mathcal{A}_{\bullet}\right) \subset \mathcal{D}^{b}\left(\mathcal{A}_{\bullet}\right)$ is fully faithful, and the famous theorem of Serre provides equivalences of categories

$$
\mathcal{A}_{\bullet}-\bmod / \mathcal{A}_{\bullet}-\bmod _{\text {tors }} \cong \operatorname{Coh}(Y), \quad \mathcal{D}^{b}\left(\mathcal{A}_{\bullet}\right) / \mathcal{D}_{\text {tors }}^{b}\left(\mathcal{A}_{\bullet}\right) \cong \mathcal{D}^{b}(Y),
$$


where $\operatorname{Coh}(Y)$ is the abelian category of coherent sheaves of $\mathcal{O}_{Y}$-modules, and $\mathcal{D}^{b}(Y)$ is its bounded derived category ${ }^{1}$. Moreover, let us denote by $\bar{f}^{*}: \mathcal{A} .-\bmod \rightarrow \operatorname{Coh}(Y)$ the natural projection; then it has a right-adjoint $\bar{f}_{*}: \operatorname{Coh}(Y) \rightarrow \mathcal{A}$.-mod given by

$$
\left(\bar{f}_{*} \mathcal{E}\right)_{n}=f_{*} \mathcal{E}(n), \quad n \geq 0
$$

and its derived functor $R^{\bullet} \bar{f}_{*}: \mathcal{D}^{b}(Y) \rightarrow \mathcal{D}^{b}\left(\mathcal{A}_{\bullet}\right)$ is right-adjoint to the projection $\bar{f}^{*}: \mathcal{D}^{b}\left(\mathcal{A}_{\mathbf{e}}\right) \rightarrow \mathcal{D}^{b}(Y)$. We have $\bar{f}^{*} \circ R^{\bullet} \bar{f}_{*} \cong \mathrm{id}$, and we have a semiorthogonal decomposition

$$
\mathcal{D}^{b}\left(\mathcal{A}_{\bullet}\right)=\left\langle\mathcal{D}^{b}(Y), \mathcal{D}_{\text {tors }}^{b}\left(\mathcal{A}_{\bullet}\right)\right\rangle
$$

in the sense of $[\mathrm{BK}]$ (in other words, the projection $\mathcal{D}^{b}\left(\mathcal{A}_{\mathbf{0}}\right) \rightarrow \mathcal{D}^{b}(Y)$ induces an equivalence between the category $\mathcal{D}^{b}(Y)$ and the right orthogonal $\left.\mathcal{D}_{\text {tors }}^{b}\left(\mathcal{A}_{\bullet}\right)^{\perp} \subset \mathcal{D}^{b}\left(\mathcal{A}_{\bullet}\right)\right)$.

Assume now that we are given an ideal sheaf $\mathcal{I} \subset \mathcal{O}_{X}$, and the algebra $\mathcal{A}$. is actually given by its powers,

$$
\mathcal{A}_{n} \cong \mathcal{I}^{n}, \quad n \geq 0
$$

so that $Y$ is the blowup of $X$ in the closed subscheme $Z \subset X$ defined by the ideal sheaf $\mathcal{I}$. Here are two typical examples of the situation.

Example 1.1. If $X$ is quasiprojective and normal, and the projective map $f: Y \rightarrow X$ is birational, then we can always find an ideal sheaf $\mathcal{I} \subset \mathcal{O}_{X}$ such that (1.2) holds, so that $Y$ is automatically a blowup. Indeed, we have $\mathcal{A}_{0}=f_{*} \mathcal{O}_{Y} \cong \mathcal{O}_{X}$ by Zariski Connectedness Theorem. Moreover, we can twist the dual $\mathcal{O}_{Y}(-1)$ to a relative very ample line bundle $\mathcal{O}_{Y}(1)$ by a pullback of a sufficiently ample line bundle on $X$ so that it acquires a global section - in other word, we have an injective map $u: \mathcal{O}_{Y}(1) \rightarrow \mathcal{O}_{Y}$. Then $f_{*}(u): \mathcal{A}_{1} \rightarrow \mathcal{A}_{0}=\mathcal{O}_{X}$ identifies $\mathcal{A}_{1}$ with an ideal sheaf $\mathcal{I} \subset \mathcal{O}_{X}$, and for any $n \geq 2, f_{*}\left(u^{n}\right): \mathcal{A}_{n} \rightarrow \mathcal{A}_{0}$ identifies $\mathcal{A}_{n}$ with the $n$-th power $\mathcal{I}^{n} \subset \mathcal{O}_{X}$.

Example 1.2. Conversely, assume that the ideal sheaf $\mathcal{I} \subset X$ is nilpotent. Then the blowup $Y$ is perfectly well defined but empty.

\footnotetext{
${ }^{1}$ The abelian case is $[S]$. The derived case seems to be folklore, but see [O, Lemma 14, 15].
} 
Definition 1.3. A $Z$-filtered sheaf $\left\langle\mathcal{E}, F^{\bullet}\right\rangle$ is a coherent sheaf $\mathcal{E}$ of $\mathcal{O}_{X^{-}}$ modules equipped with a decreasing filtration by sheaves of $\mathcal{O}_{X}$-submodules $F^{n} \mathcal{E}, n \geq 0$, such that $\mathcal{I} \cdot F^{n} \mathcal{E} \subset F^{n+1} \mathcal{E}$ for any $n \geq 0$.

For any $Z$-filtered sheaf $\left\langle\mathcal{E}, F^{\bullet}\right\rangle$ and any $n, m \geq 0$, we then have $\mathcal{I}^{m}$. $F^{n} \mathcal{E} \subset F^{n+m} \mathcal{E}$, and the direct sum

$$
\mathcal{E}_{.}=\bigoplus_{n \geq 0} F^{n} \mathcal{E}
$$

is a graded module over $\mathcal{A}$. $=\bigoplus \mathcal{I}^{n}$. Denote by $\mathcal{A}$-filt the category of $Z$ filtered sheaves $\left\langle\mathcal{E}, F^{\bullet}\right\rangle$ such that $\mathcal{E}$. is locally finitely generated as a sheaf of $\mathcal{A}$.-modules (here $\mathcal{A}$ stands for the filtered algebra sheaf $\left\langle\mathcal{O}_{X}, \mathcal{I}^{\bullet}\right\rangle$, that is, $\mathcal{O}_{X}$ equipped with the $\mathcal{I}$-adic filtration). Then $\mathcal{A}$-filt is an exact category, and we can consider its derived category $\mathcal{D} F^{b}(\mathcal{A})$. Explicitly, say that a map $f$ of complexes of objects in $\mathcal{A}$-filt is a filtered quasiisomorphism if the associated graded map $\operatorname{gr}^{\bullet}(f)$ is a quasiisomorphism; then $\mathcal{D} F^{b}(\mathcal{A})$ can be obtained by inverting filtered quasiisomorphisms in the category of bounded complexes in $\mathcal{A}$-filt, as in [BBD]. There is also a description in terms of graded modules over the so-called extended Rees algebra that we recall in the beginning of Section 2.

For any locally free coherent sheaf $\mathcal{E}$ of $\mathcal{O}_{X}$-modules, equipping $\mathcal{E}$ with the filtration $F^{n} \mathcal{E}=\mathcal{I}^{n} \cdot \mathcal{E} \subset \mathcal{E}$ turns it into a $Z$-filtered sheaf. The corresponding graded module $\mathcal{E}$. is finitely generated (in fact, it is generated by its degree-0 component), so that we obtain a natural functor

$$
\mathrm{Fl}(X) \rightarrow \mathcal{A} \text {-filt. }
$$

This functor is obviously exact, so that it induces a functor

$$
\rho: \mathcal{D}^{p f}(X)=\mathcal{D}^{b}(\mathrm{Fl}(X)) \rightarrow \mathcal{D} F^{b}(\mathcal{A})
$$

Lemma 1.4. The functor $\rho$ of (1.5) is fully faithful.

Proof. Let $\mathcal{A}$-filt ${ }_{f l} \subset \mathcal{A}$-filt be the full subcategory spanned by filtered sheaves $\left\langle\mathcal{E}, F^{\bullet}\right\rangle$ with $\mathcal{E} \in \mathrm{Fl}(X)$. This subcategory is closed under extensions, so that it is an exact subcategory, and its derived category $\mathcal{D} F_{f l}^{b}(\mathcal{A})$ is a full subcategory in $\mathcal{D} F^{b}(\mathcal{A})$. The embedding (1.4) obviously sends $\operatorname{Fl}(X)$ into 
$\mathcal{A}$-filt ${ }_{f l} \subset \mathcal{A}$-filt, and the forgetful functor

$$
\mathcal{A} \text {-filt } f l \rightarrow \mathrm{Fl}(X)
$$

sending $\left\langle\mathcal{E}, F^{\bullet}\right\rangle$ to $\mathcal{E}$ is right-adjoint to the embedding (1.4). Since the composition of (1.4) and (1.6) is the identity functor, (1.4) is fully faithful. Moreover, (1.6) is exact, thus induces a functor $\mathcal{D} F_{f l}^{b}(\mathcal{A}) \rightarrow \mathcal{D}^{p f}(X)$ rightadjoint to (1.4), and its composition with the functor $\rho$ of (1.5) is again isomorphic to the identity functor, so that $\rho$ is fully faithful.

We can now formulate our results. Denote by $\mathcal{D} F_{\text {tors }}^{b}(\mathcal{A}) \subset \mathcal{D} F^{b}(\mathcal{A})$ the full subcategory spanned by complexes of modules $\mathcal{E}$ such that $F^{n} \mathcal{E}=0$ for $n \gg 0$. Then sending $\left\langle\mathcal{E}, F^{\bullet}\right\rangle$ to $\mathcal{E}$. of $(1.3)$ gives a functor $\mathcal{A}$-filt $\rightarrow \mathcal{A}$.-mod. This functor extends to a functor

$$
\mathcal{D} F^{b}(\mathcal{A}) \rightarrow \mathcal{D}^{b}\left(\mathcal{A}_{\bullet}\right)
$$

sending $\mathcal{D} F_{\text {tors }}^{b}(\mathcal{A})$ into $\mathcal{D}_{\text {tors }}^{b}(\mathcal{A}$.$) .$

Lemma 1.5. The functor (1.7) induces an equivalence of categories

$$
\mathcal{D} F^{b}(\mathcal{A}) / \mathcal{D} F_{\text {tors }}^{b}(\mathcal{A}) \cong \mathcal{D}^{b}\left(\mathcal{A}_{\bullet}\right) / \mathcal{D}_{\text {tors }}^{b}\left(\mathcal{A}_{\bullet}\right) \cong \mathcal{D}^{b}(Y)
$$

and we have a semiorthogonal decomposition

$$
\mathcal{D} F^{b}(\mathcal{A})=\left\langle\mathcal{D}^{b}(Y), \mathcal{D} F_{\text {tors }}^{b}(\mathcal{A})\right\rangle
$$

Moreover, say that a $Z$-filtered module $\left\langle\mathcal{E}, F^{\bullet}\right\rangle$ is $n$-stable for some $n \geq 0$ if $F^{n} \mathcal{E}=\mathcal{E}$, let $\mathcal{D} F^{b}(\mathcal{A})_{n} \subset \mathcal{D} F^{b}(\mathcal{A})$ be the full subcategory spanned by complexes of $n$-stable sheaves, and let

$$
\mathcal{D} F_{\text {tors }}^{b}(\mathcal{A})_{n}=\mathcal{D} F_{\text {tors }}^{b}(\mathcal{A}) \cap \mathcal{D} F^{b}(\mathcal{A})_{n}
$$

Lemma 1.6. For any $n \geq 0$, we have a natural equivalence of categories

$$
\mathcal{D} F_{\text {tors }}^{b}(\mathcal{A})_{n} / \mathcal{D} F_{\text {tors }}^{b}(\mathcal{A})_{n+1} \cong \mathcal{D}^{b}(Z)
$$

and the projection $\mathcal{D} F_{\text {tors }}^{b}(\mathcal{A})_{n} \rightarrow \mathcal{D}^{b}(Z)$ has a right-adjoint, so that we have a semiorthogonal decomposition

$$
\mathcal{D} F_{\text {tors }}^{b}(\mathcal{A})_{n}=\left\langle\mathcal{D}^{b}(Z), \mathcal{D} F_{\text {tors }}^{b}(\mathcal{A})_{n+1}\right\rangle
$$


Definition 1.7. For any integer $n \geq 0$, the $n$-refined blowup of $X$ in $Z \subset X$ is the quotient category

$$
\operatorname{Bl}_{n}(X, Z)=\mathcal{D} F^{b}(\mathcal{A}) / \mathcal{D} F_{\text {tors }}^{b}(\mathcal{A})_{n}
$$

Note that Lemma 1.5 and Lemma 1.6 imply that we have a semiorthogonal decomposition

$$
\mathcal{D} F^{b}(\mathcal{A})=\left\langle\operatorname{Bl}_{n}(X, Z), \mathcal{D} F_{\text {tors }}^{b}(\mathcal{A})_{n}\right\rangle
$$

Proposition 1.8. For $n \gg 0$, the functor

$$
\rho_{n}: \mathcal{D}^{p f}(X) \rightarrow \operatorname{Bl}_{n}(X, Z)=\mathcal{D} F^{b}(\mathcal{A}) / \mathcal{D} F_{\text {tors }}^{b}(\mathcal{A})_{n}
$$

obtained by composing the fully faithful embedding $\rho$ of (1.5) with the natural projection is fully faithful.

We note that by Lemma 1.5 , the 0 -refined blowup $\mathrm{Bl}_{0}(X, Z)$ is just the category $\mathcal{D}^{b}(Y)$. The functor $\rho_{0}$ then coincides with the natural pullback functor

$$
f^{*}: \mathcal{D}^{p f}(X) \rightarrow \mathcal{D}^{b}(Y)
$$

If $X$ has rational singularities, then already this functor is fully faithful, and we have a categorical resolution even without further refinements.

In the general case, however, we do need to take some $n \geq 1$ (see Remark 2.2 for an effective lower bound on $n$ ). By Lemma 1.6, for any such $n$, the $n$-refined blowup has a natural decreasing filtration by full subcategories whose top quotient is $\mathcal{D}^{b}(Y)$, and the other quotients are equivalent to $\mathcal{D}^{b}(Z)$. Moreover, we in fact have a semiorthogonal decomposition

$$
\mathrm{Bl}_{n}(X, Z)=\left\langle\mathcal{D}^{b}(Y), \mathcal{D}^{b}(Z), \ldots, \mathcal{D}^{b}(Z)\right\rangle,
$$

with $n$ copies of $\mathcal{D}^{b}(Z)$ in the right-hand side. Proposition 1.8 then shows that for a large enough $n$, the $n$-refined blowup gives a "partial categorical resolution" of $X$ in an appropriate sense. Note that in the situation of Example 1.2, $\mathcal{D}^{b}(Y)$ is empty. However, Proposition 1.8 still works; in this case, it coincides with the Auslander construction that forms Step 2 in the categorical resolution procedure of $[\mathrm{KL}]$.

Remark 1.9. One might be tempted to avoid the need for Proposition 1.8 by saying that $\mathcal{D} F^{b}(\mathcal{A})$ itself is a categorical resolution of $\mathcal{D}^{p f}(X)$. Indeed, 
the functor $\rho: \mathcal{D}^{p f}(X) \rightarrow \mathcal{D} F^{b}(\mathcal{A})$ is fully faithful by Lemma 1.4, and it can be shown that $\mathcal{D} F^{b}(\mathcal{A})$ admits a version of the semiorthogonal decomposition (1.9) with infinitely many copies of $\mathcal{D}^{b}(Z)$. However, such "infinite" semiorthogonal decompositions seem to be useless; in practice, it is essential that we only have a finite number of terms.

\section{Proofs}

As in Section 1, fix a Noetherian scheme $X$ with an ideal sheaf $\mathcal{I} \subset \mathcal{O}_{X}$, assume that $\operatorname{Coh}(X)$ has enough locally free sheaves, let $Z \subset X$ be the closed subscheme defined by $\mathcal{I}$, let

$$
\mathcal{A}_{.}=\bigoplus_{n \geq 0} \mathcal{I}^{n}
$$

and let $Y=\operatorname{Proj} \mathcal{A}_{\mathbf{e}}$. It is convenient to consider the extended Rees algebra $\widetilde{\mathcal{A}}$. associated to the $\mathcal{I}$-adic filtration on $\mathcal{O}_{X}$. This is a graded subalgebra $\widetilde{\mathcal{A}} . \subset \mathcal{O}_{X}\left[u, u^{-1}\right]$ in the algebra $\mathcal{O}_{X}\left[u, u^{-1}\right]$ of Laurent polynomials in one variable $u$ of degree -1 , and it is given by

$$
\widetilde{\mathcal{A}}_{n}= \begin{cases}\mathcal{I}^{n}, & n \geq 1, \\ \mathcal{O}_{X}, & n \leq 0\end{cases}
$$

Alternatively, $\widetilde{\mathcal{A}}$. is the quotient of the polynomial algebra $\mathcal{A}_{\bullet}[u]$ by the relations $a \cdot u=u(a)$ for any $a \in \mathcal{A}_{1}$, where $u: \mathcal{A}_{1}=\mathcal{I} \rightarrow \mathcal{A}_{0}=\mathcal{O}_{X}$ is the natural embedding. Yet another description is

$$
\widetilde{\mathcal{A}}_{\bullet}=\mathcal{A}_{\bullet} \oplus u \mathcal{A}_{0}[u]
$$

with the product given again by $a \cdot u=u(a)$. In particular, we have an algebra embedding $\mathcal{A}$. $\rightarrow \widetilde{\mathcal{A}}$. that identifies $\mathcal{A}$. with the subalgebra of elements in $\widetilde{\mathcal{A}}$. of non-negative degree.

The category $\widetilde{\mathcal{A}}$.-mod of graded sheaves of $\widetilde{\mathcal{A}}$.-modules locally finitely generated over $\widetilde{\mathcal{A}}$. is then an abelian category, with the bounded derived category $\mathcal{D}^{b}\left(\widetilde{\mathcal{A}}_{\mathbf{0}}\right)$. Say that $\mathcal{E}_{\mathbf{0}} \in \widetilde{\mathcal{A}}_{\mathbf{0}}$-mod is $n$-stable for some integer $n$ if $u: \mathcal{E}_{i} \rightarrow \mathcal{E}_{i-1}$ is an isomorphism for $i \leq n$, let $\widetilde{\mathcal{A}} \cdot-\bmod _{n} \subset \widetilde{\mathcal{A}}$ - $-\bmod$ be the full subcategory spanned by $n$-stable modules, and let $\mathcal{D}_{n}^{b}\left(\widetilde{\mathcal{A}}_{\bullet}\right) \subset \mathcal{D}^{b}\left(\widetilde{\mathcal{A}}_{\bullet}\right)$ be its bounded derived category. Then for any $Z$-filtered sheaf $\left\langle\mathcal{E}, F^{\bullet}\right\rangle \in \mathcal{A}$-filt, the graded sheaf $\mathcal{E}$. of (1.3) extends naturally to a 0 -stable graded module 
$\widetilde{\mathcal{E}} . \in \widetilde{\mathcal{A}}_{\bullet}$-mod given by

$$
\widetilde{\mathcal{E}}_{n}= \begin{cases}F^{n} \mathcal{E}, & n \geq 1 \\ \mathcal{E}, & n \leq 0\end{cases}
$$

with $u: \widetilde{\mathcal{E}}_{\bullet} \rightarrow \widetilde{\mathcal{E}}_{\bullet}-1$ acting by the natural embedding. This defines an exact functor $\mathcal{A}$-filt $\rightarrow \widetilde{\mathcal{A}}$.-mod that identifies $\mathcal{A}$-filt with the full subcategory in $\widetilde{\mathcal{A}}$.-mod spanned by 0 -stable modules $\mathcal{E}$. $\in \widetilde{\mathcal{A}}_{\text {. }}$-mod such that the map $u$ : $\mathcal{E}_{i} \rightarrow \mathcal{E}_{i-1}$ is injective for any $i$. The whole category $\widetilde{\mathcal{A}}$.-mod is then the abelian envelope of the exact (quasiabelian) category $\mathcal{A}$-filt, see e.g. [Sch], so that on the level of derived categories, the corresponding functor

$$
\mathcal{D} F^{b}(\mathcal{A}) \rightarrow \mathcal{D}_{0}^{b}\left(\widetilde{\mathcal{A}}_{\bullet}\right)
$$

is an equivalence of categories. For any $n \geq 1$, it sends $n$-stable $Z$-filtered modules to $n$-stable $\widetilde{\mathcal{A}}$.-modules and identifies $\mathcal{D} F^{b}(\mathcal{A})_{n}$ with $\mathcal{D}_{n}^{b}(\widetilde{\mathcal{A}}$. $)$. Under the equivalence (2.1), the functor $\rho$ of (1.5) sends $\mathcal{E} \in \operatorname{Fl}(X)$ to $\mathcal{E} \otimes \mathcal{O}_{X} \widetilde{\mathcal{A}}_{\text {. }}$. The functor

$$
\tau: \mathcal{D}_{0}^{b}\left(\tilde{\mathcal{A}}_{\bullet}\right) \rightarrow \mathcal{D}^{b}(\mathcal{A})
$$

corresponding to (1.7) is induced by the exact forgetful functor

$$
\tau: \widetilde{\mathcal{A}} \cdot-\bmod _{0} \rightarrow \mathcal{A} \cdot-\bmod
$$

forgetting the negative degree components and the action of the generator $u \in \widetilde{\mathcal{A}}_{\text {. }}$.

Proof of Lemma 1.5. Note that the functor $\bar{f}_{*}: \operatorname{Coh}(Y) \rightarrow \mathcal{A}_{\mathbf{0}}-\bmod$ of (1.1) factors through the projection $\tau$ of (2.2). Indeed, the natural maps $u$ : $\mathcal{O}_{Y}(n+1) \rightarrow \mathcal{O}_{Y}(n), n \geq 0$ induce maps

$$
u:\left(\bar{f}_{*} \mathcal{E}\right)_{n+1} \rightarrow\left(\bar{f}_{*} \mathcal{E}\right)_{n}
$$

for any $\mathcal{E} \in \operatorname{Coh}(Y)$, so that if we set

$$
\tilde{f}_{*}(\mathcal{E})_{n}= \begin{cases}\bar{f}_{*}(\mathcal{E})_{n}, & n \geq 1 \\ \bar{f}_{*}(\mathcal{E})_{0}, & n \leq 0\end{cases}
$$

then the natural $\mathcal{A}$.-action on $\bar{f}_{*}(\mathcal{E})$ and the maps $(2.3)$ equip the graded sheaf $\widetilde{f}_{*}(\mathcal{E})$. with an action of the extended Rees algebra $\widetilde{\mathcal{A}}_{.}$. Thus we obtain 
a functor

$$
\widetilde{f}_{*}: \operatorname{Coh}(Y) \rightarrow \widetilde{\mathcal{A}}_{\bullet}-\bmod _{0}
$$

such that $\bar{f}_{*} \cong \tau \circ \widetilde{f}_{*}$, where $\tau$ is the forgetful functor (2.2).

Moreover, for any $\mathcal{E}_{\bullet} \in \widetilde{\mathcal{A}}_{\mathbf{0}}-\bmod _{0}$, the adjunction map $\tau\left(\mathcal{E}_{\bullet}\right) \rightarrow \bar{f}_{*} \bar{f}^{*} \tau\left(\mathcal{E}_{\bullet}\right)$ is compatible with the maps $(2.3)$, thus comes from a map $\mathcal{E}_{\bullet} \rightarrow \widetilde{f}_{*} \tilde{f}^{*} \mathcal{E}_{\bullet}$, where we denote $\widetilde{f}^{*}=\bar{f}^{*} \circ \tau$.

Passing to the derived functors, we obtain a functor

$$
R^{\bullet} \widetilde{f}_{*}: \mathcal{D}^{b}(Y) \rightarrow \mathcal{D}_{0}^{b}\left(\widetilde{\mathcal{A}}_{\mathbf{.}}\right)
$$

such that $\tau \circ R^{\bullet} \widetilde{f}_{*} \cong R^{\bullet} \bar{f}_{*}$, and a functorial map

$$
a: \mathcal{E}_{\bullet} \rightarrow R^{\bullet} \tilde{f}_{*} \tilde{f}^{*} \mathcal{E}
$$

for any $\mathcal{E}_{\bullet} \in \mathcal{D}_{0}^{b}\left(\mathcal{A}_{\bullet}\right)$. Note that we have

$$
\tilde{f}^{*} \circ R^{\bullet} \tilde{f}_{*}=\bar{f}^{*} \circ \tau \circ R^{\bullet} \tilde{f}_{*} \cong \bar{f}^{*} \circ R^{\bullet} \bar{f}_{*} \cong \mathrm{ld},
$$

and $\tau(a)$ is the adjunction map between $\bar{f}^{*}$ and $R^{\bullet} \bar{f}_{*}$. Then it is easy to check that (2.4) together with the isomorphism (2.5) define an adjunction between the functors $\widetilde{f}^{*}$ and $R^{\bullet} \widetilde{f}_{*}$, so that (2.5) implies that $R^{\bullet} \widetilde{f}^{*}$ is a fully faithful embedding onto a left-admissible subcategory. To finish the proof, it suffices to notice that the kernel of the functor

$$
\tilde{f}^{*}: \mathcal{D} F^{b}(\mathcal{A}) \cong \mathcal{D}_{0}^{b}\left(\widetilde{\mathcal{A}}_{\bullet}\right) \rightarrow \mathcal{D}^{b}(Y)
$$

is exactly the subcategory $\mathcal{D} F_{\text {tors }}^{b}(\mathcal{A}) \subset \mathcal{D} F^{b}(\mathcal{A})$.

Proof of Lemma 1.6. Denote by $i: Z \rightarrow X$ the embedding. For any coherent sheaf $\mathcal{E}$ on $Z, i_{*} \mathcal{E}$ with the filtration $F^{n} i_{*} \mathcal{E}=i_{*} \mathcal{E}, F^{n+1} i_{*} \mathcal{E}=0$ is an $n$-stable $Z$-filtered sheaf on $X$. This gives a fully faithful exact functor $i_{n}: \operatorname{Coh}(Z) \rightarrow$ $\mathcal{A}$-filt ${ }_{n}$ to the full subcategory $\mathcal{A}$-filt ${ }_{n} \subset \mathcal{A}$-filt spanned by $n$-stable $Z$-filtered sheaves.

The functor $\operatorname{gr}_{F}^{n}: \mathcal{A}$-filt ${ }_{n} \rightarrow \operatorname{Coh}(Z)$ sending a $Z$-filtered sheaf to its $n$-th associated graded quotient with respect to the filtration is obviously leftadjoint to the functor $i_{n}$. The functors $i_{n}$ and $\operatorname{gr}_{F}^{n}$ then extend to an adjoint pair of functors between the categories of complexes of objects in the exact categories $\mathcal{A}$-filt ${ }_{n}, \operatorname{Coh}(Z)$. Moreover, $i_{n}$ is exact, and the functor $\operatorname{gr}_{F}^{n}$ is by definition also exact with respect to the exact structure on $\mathcal{A}$-filt, that is, 
it sends filtered quasiisomorphisms between filtered complexes to quasiisomorphisms. Thus $i_{*}$ and $\operatorname{gr}_{F}^{n}$ descend to an adjoint pair of functors

$$
i_{n}: \mathcal{D}^{b}(Z) \rightarrow \mathcal{D} F^{b}(\mathcal{A})_{n}, \quad \operatorname{gr}_{F}^{n}: \mathcal{D} F^{b}(\mathcal{A})_{n} \rightarrow \mathcal{D}^{b}(Z)
$$

between derived categories, and the isomorphism $\operatorname{gr}_{F}^{n} \circ i_{n} \cong \mathrm{Id}$ on the level of complexes induces an analogous isomorphism on the level of derived categories. Therefore $i_{n}: \mathcal{D}^{b}(Z) \rightarrow \mathcal{D} F^{b}(\mathcal{A})_{n}$ is fully faithful. It remains to notice that $i_{n}$ sends $\mathcal{D}^{b}(Z)$ into $\mathcal{D} F_{\text {tors }}^{b}(\mathcal{A})_{n} \subset \mathcal{D} F^{b}(\mathcal{A})_{n}$, and the orthogonal ${ }^{\perp} i_{n}\left(\mathcal{D}^{b}(Z)\right) \subset \mathcal{D} F_{\text {tors }}^{b}(\mathcal{A})_{n}$ — that is, the kernel of the functor $\operatorname{gr}_{F}^{n}$ — is exactly $\mathcal{D} F_{\text {tors }}^{b}(\mathcal{A})_{n+1}$.

Remark 2.1. In particular, for $n=0$, the proof of Lemma 1.6 shows that the functor

$$
i_{0}: \mathcal{D}^{b}(Z) \rightarrow \mathcal{D} F^{b}(\mathcal{A})
$$

is fully faithful. Note that if we compose $i_{0}$ with the tautological forgetful functor $\mathcal{D} F^{b}(\mathcal{A}) \rightarrow \mathcal{D}^{b}(X),\left\langle\mathcal{E}, F^{\bullet}\right\rangle \mapsto \mathcal{E}$, then the composition is the direct image functor $i_{*}$ with respect to the embedding $i: Z \rightarrow X$, and it certainly is not fully faithful on the level of derived categories. Thus working with filtered derived categories is crucial for the construction.

Before we prove Proposition 1.8, let us make one observation. Lemma 1.5 provides a natural quotient functor

$$
\tilde{f}^{*}: \mathcal{D} F^{b}(\mathcal{A}) \rightarrow \mathcal{D} F^{b}(\mathcal{A}) / \mathcal{D} F_{\text {tors }}^{b}(\mathcal{A}) \cong \mathcal{D}^{b}(Y)
$$

and as noted in Section 1, the composition $\rho_{0}=\tilde{f}^{*} \circ \rho: \mathcal{D}^{p f}(X) \rightarrow \mathcal{D}^{b}(Y)$ is isomorphic to the pullback functor $f^{*}$ of (1.8). More generally, for any object $\mathcal{E} \in \mathrm{Fl}(X)$ and any filtered complex $\mathcal{F} \in \mathcal{D} F^{b}(\mathcal{A})$, the tensor product $\mathcal{E} \otimes_{\mathcal{O}_{X}} \mathcal{F}$ is naturally a filtered complex, and we have a natural functorial identification

$$
\tilde{f}^{*}\left(\mathcal{E} \otimes_{\mathcal{O}_{X}} \mathcal{F}\right) \cong f^{*} \mathcal{E} \otimes_{\mathcal{O}_{Y}} \tilde{f}^{*} \mathcal{F}
$$

Lemma 1.5 also provides a functor $R^{\bullet} \tilde{f}_{*}: \mathcal{D}^{b}(Y) \rightarrow \mathcal{D} F^{b}(\mathcal{A})$ right-adjoint to $\widetilde{f}^{*}$, and for any $\mathcal{E} \in \operatorname{Fl}(X), \mathcal{F} \in \operatorname{Coh}(Y)$, we have a natural map

$$
\tilde{f}^{*}\left(\mathcal{E} \otimes_{\mathcal{O}_{X}} R^{\bullet} \tilde{f}_{*} \mathcal{F}\right) \stackrel{a}{\longrightarrow} f^{*} \mathcal{E} \otimes_{\mathcal{O}_{Y}} \tilde{f}^{*} R^{\bullet} \tilde{f}_{*} \mathcal{F} \stackrel{\mathrm{id} \otimes b}{\longrightarrow} f^{*} \mathcal{E} \otimes_{\mathcal{O}_{Y}} \mathcal{F}
$$


where $a$ is the isomorphism (2.6), and $b: \tilde{f}^{*} R^{\bullet} \widetilde{f}_{*} \mathcal{F} \rightarrow \mathcal{F}$ is the adjunction map. Then by adjunction, (2.7) induces a map

$$
\mathcal{E} \otimes_{\mathcal{O}_{X}} R^{\bullet} \tilde{f}_{*} \mathcal{F} \rightarrow R^{\bullet} \tilde{f}_{*}\left(\tilde{f}^{*} \mathcal{E} \otimes_{\mathcal{O}_{Y}} \mathcal{F}\right)
$$

and we observe that (2.8) is an isomorphism. Indeed, it suffices to check it separately on every filtered component, and then it immediately follows from the usual projection formula and (1.3).

Proof of Proposition 1.8. By Lemma 1.5 and Lemma 1.6, the projection $\mathcal{D} F^{b}(\mathcal{A}) \rightarrow \mathrm{Bl}_{n}(X, Z)$ actually comes from a semiorthogonal decomposition. In particular, it has a right-adjoint functor identifying $\mathrm{Bl}_{n}(X, Z)$ with the right orthogonal

$$
\mathcal{D} F_{\text {tors }}^{b}(\mathcal{A})_{n}^{\perp} \subset \mathcal{D} F^{b}(\mathcal{A}) .
$$

Since the functor $\rho$ of (1.5) is fully faithful, it suffices to prove that for $n \gg 0$, it sends the whole $\mathcal{D}^{p f}(X)$ into this orthogonal.

Consider first the object $\mathcal{O}_{X} \in \mathcal{D}^{p f}(X)$, and let $\overline{\mathcal{O}}_{X} \in \mathcal{D} F^{b}(\mathcal{A})$ be the cone of the adjunction map $\rho\left(\mathcal{O}_{X}\right) \cong \mathcal{A} \rightarrow R^{\bullet} \widetilde{f}_{*} \widetilde{f}^{*} \mathcal{A} \cong R^{\bullet} \widetilde{f}_{*} \mathcal{O}_{Y}$. We then have a distinguished triangle

$$
\mathcal{A} \longrightarrow R^{\bullet} \tilde{f}_{*} \mathcal{O}_{Y} \longrightarrow \overline{\mathcal{O}_{X}} \longrightarrow \mathcal{A}[1]
$$

and by definition, $\overline{\mathcal{O}}_{X}$ lies inside $\mathcal{D} F_{\text {tors }}^{b}(\mathcal{A}) \subset \mathcal{D} F^{b}(\mathcal{A})$, so that $\operatorname{gr}_{F}^{n} \overline{\mathcal{O}}_{X}=0$ for $n \geq N$ for some fixed constant $N \geq 0$.

Now for every $\mathcal{E} \in \operatorname{Fl}(X)$, the product $\mathcal{E} \otimes_{\mathcal{O}_{X}} \overline{\mathcal{O}}_{X}$ also lies in the subcategory $\mathcal{D} F_{\text {tors }}^{b}(\mathcal{A}) \subset \mathcal{D} F^{b}(\mathcal{A})$, and we have

$$
\operatorname{gr}_{F}^{n}\left(\mathcal{E} \otimes_{\mathcal{O}_{X}} \overline{\mathcal{O}}_{X}\right) \cong \mathcal{E} \otimes_{\mathcal{O}_{X}} \operatorname{gr}_{F}^{n} \overline{\mathcal{O}}_{X}=0
$$

for $n \geq N$. By Lemma 1.6, this implies that $\mathcal{E} \otimes_{\mathcal{O}_{X}} \overline{\mathcal{O}}_{X}$ lies in

$$
\mathcal{D} F_{\text {tors }}^{b}(\mathcal{A})_{n}^{\perp} \subset \mathcal{D} F_{\text {tors }}^{b}(\mathcal{A}) \subset \mathcal{D} F^{b}(\mathcal{A}) \text {. }
$$

On the other hand, by $(2.8)$, the product $\mathcal{E} \otimes_{\mathcal{O}_{X}} R^{\bullet} \tilde{f}_{*} \mathcal{O}_{Y}$ lies in the image of the fully faithful embedding $R \cdot \widetilde{f}_{*}$. Therefore it is orthogonal to the category $\mathcal{D} F_{\text {tors }}^{b}(\mathcal{A})$ containing $\mathcal{D} F_{\text {tors }}^{b}(\mathcal{A})_{n}$, that is, also lies in $\mathcal{D} F_{\text {tors }}^{b}(\mathcal{A})_{n}^{\perp}$.

Tensoring $\mathcal{E}$ with the exact triangle $(2.9)$, we deduce that for any object $\mathcal{E} \in \operatorname{Fl}(X)$, hence also for any $\mathcal{E} \in \mathcal{D}^{p f}(X)$, the object $\rho(\mathcal{E})=\mathcal{E} \otimes_{\mathcal{O}_{X}} \mathcal{A}$ lies in $\mathcal{D} F_{\text {tors }}^{b}(\mathcal{A})_{n}^{\perp}$ as soon as $n \geq N$. 
Remark 2.2. As we see from the proof, the constant $n$ in Proposition 1.8 admits an effective lower bound: it is necessary and sufficient that

$$
R^{\bullet} \mathcal{O}_{Y}(m) \cong \mathcal{I}^{m}
$$

for any $m \geq n$.

We conclude with remarking that throughout the paper, the actual geometry of the schemes $X$ and $Y$ has been used very little in either the statements or the proofs. We in fact only need it to control the size of things - namely, to show that various categories of finitely generated modules are abelian, and that the adjoint functor $\bar{f}_{*}$ of (1.1) exists on the level of bounded derived categories. If we drop the finiteness and boundedness assumptions, then everything works for arbitrary sheaves of graded associative algebras, in fact even for non-commutative ones (for Lemma 1.6, the same proof goes through literally, and for Lemma 1.5 and Proposition 1.8, the arguments are very easy to generalize). One can also get by with weaker conditions on the algebras such as coherence. We did not pursue this since, as we have explained in the introduction, our main motivation is in any case geometric, and at the moment, we do not have interesting examples where larger generality is required.

Acknowledgements. This paper was originally conceived during the conference in Tokyo University celebrating the 60th anniversary of Prof. Yu. Kawamata - the second author was giving a talk on [KL], and the first author, while chairing the talk, was trying to come up with a meaningful question. In the event, there was so much interest in the talk that the question was not required, so it was only asked later and in private. The question was, is it possible to combine Step 1 and Step 2 of [KL]. The answer is the present paper. We take this opportunity to congratulate Prof. Kawamata once again, and to thank the organizers of the conference, in particular Prof. K. Oguiso, for inviting us both and for setting up this opportune moment.

\section{References}

[BBD] A. Beilinson, J. Bernstein and P. Deligne, Faisceaux Pervers. Astérisque, 100, Soc. Math. de France, 1983.

[Bl] A. Blanc, Invariants topologiques des Espaces non commutatifs. arXiv: 1307.6430 . 
[BK] A. Bondal and M. Kapranov, Representable functors, Serre functors, and reconstructions. (Russian) Izv. Akad. Nauk SSSR Ser. Mat., 53 (1989), 1183-1205, 1337; translation in Math. USSR-Izv., 35 (1990), $519-541$.

[E] A. Efimov, Homotopy finiteness of some DG categories from algebraic geometry. arXiv:1308.0135.

[Ka1] D. Kaledin, Non-commutative Hodge-to-de Rham degeneration via the method of Deligne-Illusie. Pure Appl. Math. Q., 4 (2008), 785875 .

[Ka2] D. Kaledin, Motivic structures in non-commutative geometry. Proc. ICM 2010.

[Ke1] B. Keller, Derived categories and universal problems. Communications in Algebra, 19 (1991), 699-747.

[Ke2] B. Keller, On differential graded categories. In: International Congress of Mathematicians, Vol. II, Eur. Math. Soc., Zürich, 2006, 151-190.

[KL] A. Kuznetsov and V. Lunts, Categorical resolutions of irrational singularities, Int. Math. Res. Not., 13 (2015), 4536-4625.

[L] V. Lunts, Categorical resolution of singularities. J. Algebra, 323 (2010), 2977-3003.

[O] D. Orlov, Derived categories of coherent sheaves and triangulated categories of singularities. In: Algebra, Arithmetic, and Geometry, Birkhäuser Boston, 2009, 503-531.

[R] R. Rouquier, Dimensions of triangulated categories. Journal of Ktheory: K-theory and its Applications to Algebra, Geometry, and Topology (1), 02 (2008), 193-256.

[Sch] J.-P. Schneiders, Quasi-abelian categories and sheaves. Société mathématique de France, 1999.

[S] J.-P. Serre, Faisceaux algébriques coherents. Ann. of Math., 61 (1955), 197-278.

[TV] B. Toën and M. Vaquié, Moduli of objects in dg-categories. Ann. Sci. École Norm. Sup. (4), 40 (2007), 387-444. 
Steklov Math Institute, Algebraic Geometry section, Moscow, Russia \& Laboratory of Algebraic Geometry, National Research University Higher School of Economics, Russian Federation \& Center for Geometry and Physics, iBs, Pohang, Rep. of Korea E-mail address: kaledin@mi.ras.ru

Steklov Math Institute, Algebraic Geometry section, Moscow, Russia \& The Poncelet Laboratory, Independent University of Moscow \& Laboratory of Algebraic Geometry, National Research University Higher School of Economics, Russian Federation E-mail address: akuznet@mi.ras.ru

Received March 18, 2015 